\title{
Insight into Applications of Deep Learning
}

\author{
AishwaryA T*, Ravi Kumar V \\ Dept of CSE, Vidyavardhaka College Of Engineering, Mysuru
}

DOI: https://doi.org/10.21467/proceedings.1.76

* Corresponding author email: aishwaryats95@gmail.com

\begin{abstract}
Deep learning also known as deep structured learning is an element of an extended family of Machine learning. Deep learning has progressed rapidly and now demonstrates an high level of development and performance in numerous fields. Deep learning, which has evolved from the acquisition of big data, the influence of parallel, distributed computing and elegant, sophisticated training algorithms, has expedited significant advances in umpteen domains for instance Bio-informatics, Speech recognition, Image recognition, Sentiment analysis, Customer relationship management, Political leaning and Natural language processing.
\end{abstract}

Index terms: Deep learning, Neural networks, Bio-informatics, Speech recognition, Image recognition, Customer relationship management.

\section{INTRODUCTION}

Deep Learning is a novel and unhackneyed area of Machine learning research, that has been instituted with the intention of moving Machine learning near to one of its original goals: The mighty - Artificial intelligence. The triumph of Deep learning has been a catalyst to solving progressively complex machine learning problems. Deep Learning procedures and architectures have reached appealing results and also evoked admiration, allowing breakthrough improvements in performance in many difficult tasks, such as speech recognition, end-to- end machine translation, image classification or understanding etc [1]. The major basis why deep learning has convinced and lured the notice of so many explorator and engineers is because it can apprehend features and identify patterns in ways many once thought unattainable for computers. The breakthrough was epitomized by the emergence of AlphaGo [2]. Before the success of AlphaGo, experts had thought that the abstract strategical thoughts and theories that a human Go player develops over time through training and experience, were not mimicable by a computer that is, without some technological innovation and improvement. AlphaGo proved that deep learning is indeed that breakthrough.

Deep Artificial Neural Networks have obtained enormous significance in Pattern recognition and and also in Machine learning. With the advent of Neural networks, the system can solely identify patterns in the data to learn how to carry out a job. Tasks and capabilities that were

(C) 2018 Copyright held by the author(s). Published by AIJR Publisher in Proceedings of the $3^{\text {rd }}$ National Conference on Image Processing, Computing, Communication, Networking and Data Analytics (NCICCNDA 2018), April $28,2018$.

This is an open access article under Creative Commons Attribution-NonCommercial 4.0 International (CC BY-NC 4.0) license, which permits any non-commercial use, distribution, adaptation, and reproduction in any medium, as long as the original work is properly cited. ISBN: 978-81-936820-0-5 
once contemplated the realm of humans are now being executed by Deep Neural Networks. In the aeon of big data, conversion of biomedical big data into valuable knowledge has been one of the most salient and confronting challenges in bioinformatics [3]. Customer relationship management is a terminology that refers to practices, procedures and policies that companies handle and examines customer correspondence and data over the entire customer lifecycle, with the aim of bettering business relationships with customers, also assisting in customer retention and increasing sales growth.

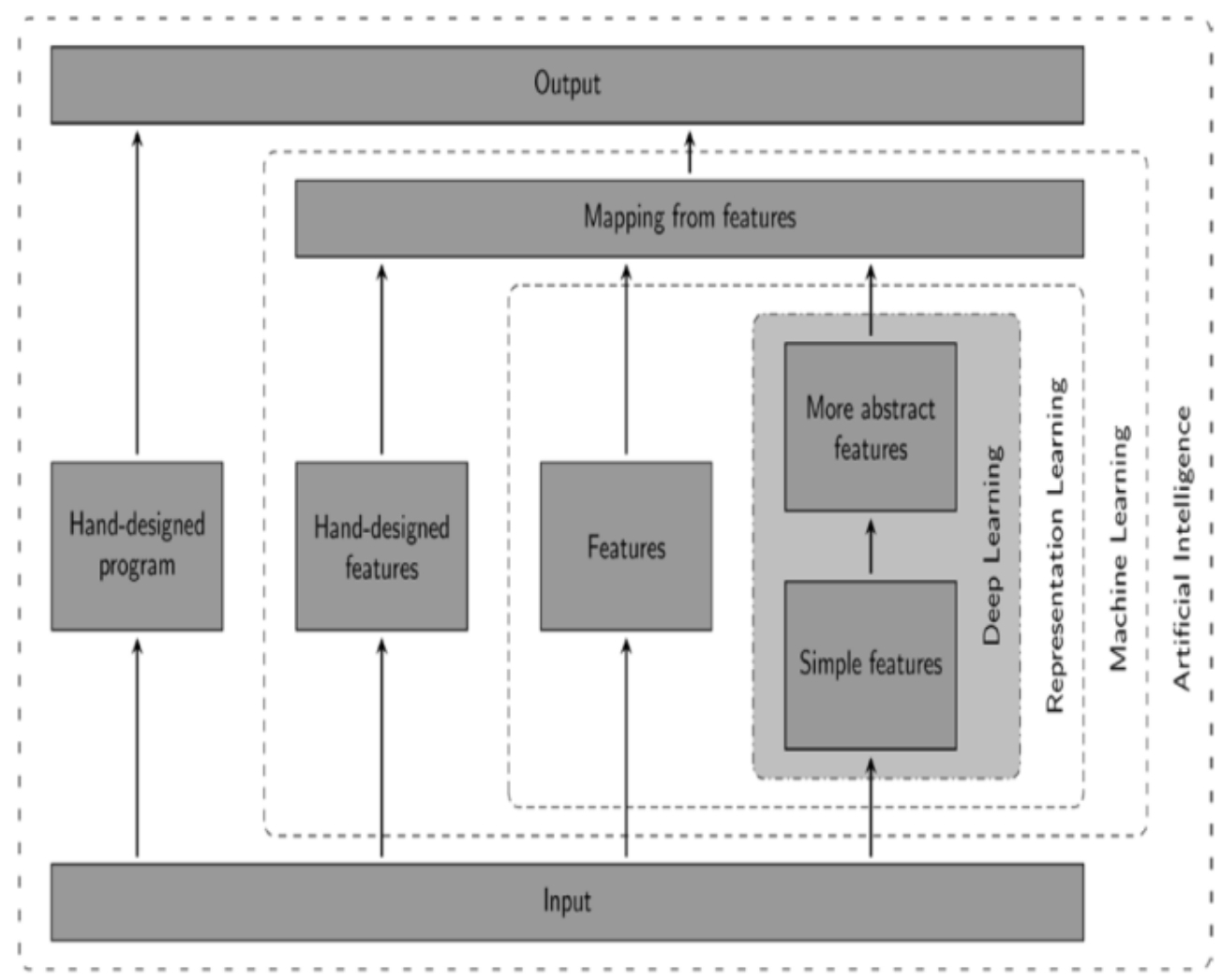

Figure 1: Relationships and high level schematics of various disciplines.

Figure.1 represents the relationship and schematics of numerous disciplines such as Deep learning, Machine learning, Representation learning and Artificial intelligence. Deep learning involves deriving simple features and deeper abstract features that can also be used in Representation learning, Machine learning and Artificial intelligence. The mapping from the features is then used to obtain the final output.

Proceedings of the $3^{\text {rd }}$ National Conference on Image Processing, Computing, Communication, Networking and Data Analytics (NCICCNDA 2018) 
Machine Learning

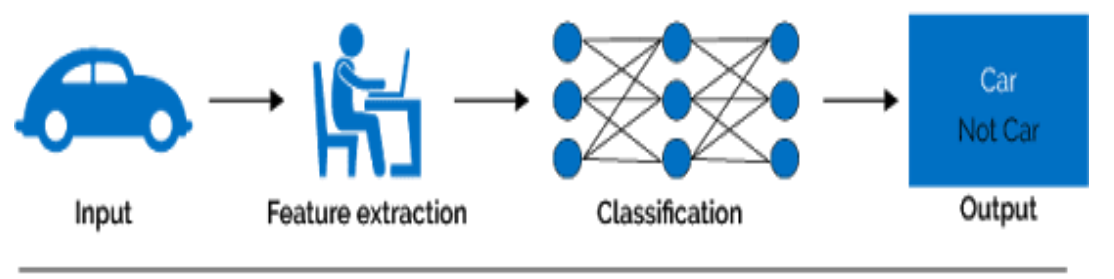

\section{Deep Learning}

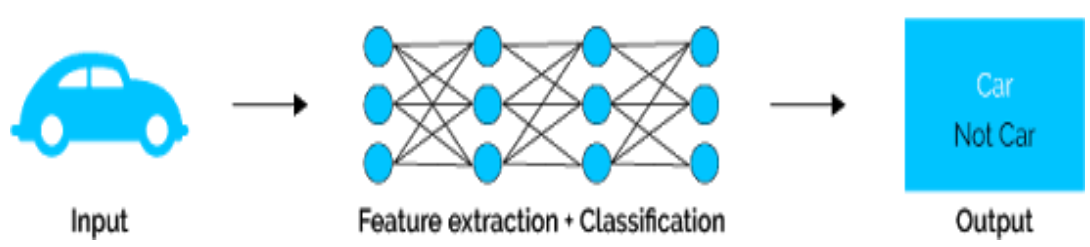

Figure 2: Machine Learning and Deep Learning

\section{DEEP LEARNING IN NEURAL NETWORKS}

Neural networks have made an extraordinary comeback in these years, after having serious concerns about the capability to train deep models, were fortuitously mellowed out by a leading - edge group of researchers who influenced the advances in algorithms, data and computing [4]. These Neural networks are demographic models inspired precisely by and partially shaped on biological neural networks. They have the ability of modelling and computing non-linear affiliations among inputs and outputs in parallel. Neural networks commonly are delineated by having adaptive weights with paths between neurons that can be accomodated by a learning algorithm that improves the model by learning from the observed data. For neural network based deep learning models, the number of layers are larger than in so referred shallow learning algorithms. Shallow algorithms are likely to be less intricate and require more straightforward understanding and learning of optimal features to use, which typically include feature selection and engineering. In comparison, Deep learning algorithms entrust on optimal ideal model selection and escalation and optimization through model tuning.

Neural networks and deep learning combines strategies, concepts and techniques from signal processing in addition to statistical techniques as well, These also include non- linear processing and transformations too. Very deep convolutional neural networks (CNN's) can be built and an earnestly low error rate for tasks like large-scale image classification can be brought about with the aid of Deep Learning. Extensive datasets have been the major reason for the rapid development of deep neural networks for visual recognition [7]. The progress and accomplishment of these systems is that they are established in the way the human brain operates and processes information all by itself, such aspect is tremendously useful for the neural network applications. 


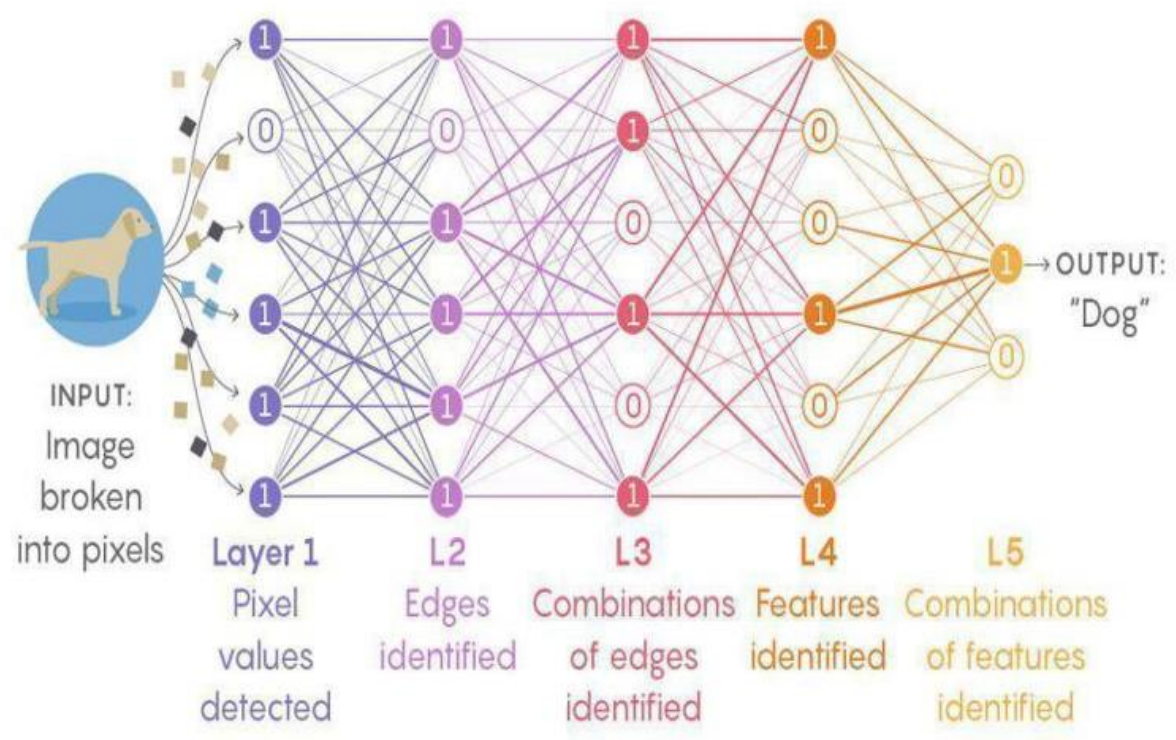

Figure 3: Deep neural networks learn by fine tuning the strengths of their connections to better conduct the signals to neurons.

Figure 3 shows the basic working of Deep Neural networks. All images that are taken as the input are broken down into a number of pixels in the first layer. The edges and combinations of edges are identified in the subsequent layers. These combinations help in obtaining features and finally provide the output based on these features recognised.

Convolutional neural network is one of the most prominent and popular type of Neural network. A CNN entwines and convolves learned features with input data and uses 2D convolutional layers. Manual feature extraction is annihilated by the CNN's and this is its important advantage.

Layer-wise relevance propagation is the most recently proposed technique. LRP is a backward propagation technique. The layer wise mapping is modelled to ensure the relevance conservation property. The LRP technique is entrenched in a conservation principle. Each neuron receives a portion of the network output and redistributes it to its predecessors in proportionate amount, until the input variables are reached.

\section{DEEP LEARNING IN BIOINFORMATICS}

Bioinformatics is an area that develops methods and software tools for perceiving biological data. Increasing the understanding of biological processes is the predominant and primary goal of Bioinformatics. Its focus on developing and applying computationally intensive techniques such as Pattern recognition, Machine learning, Deep learning and visualizations to achieve this goal, however, is what sets it apart from other approaches.

Proceedings of the $3^{\text {rd }}$ National Conference on Image Processing, Computing, Communication, Networking and Data Analytics (NCICCNDA 2018) 
Insight into Applications of Deep Learning

The decent and proper performance of conventional machine learning algorithms counts heavily on data representations called features [5]. Nevertheless, features are typically modelled and designed by human engineers with thorough domain expertise. Recognizing which features are more appropriate for the given task remains wearisome and difficult. Deep learning also facilitates Bioinformatics: finger joints can be noticed from X-ray images, lapses can be uncovered from electroencephalography (EEG) signals, splice junctions can be desclosed from DNA sequences and so on [6].

DeepSEA - A Convolutional Neural Network based algorithmic framework, performs multitask joint learning of chromatin factors (i.e., transcription factor binding, DNase I sensitivity, histone-mark profile). It is developed to prioritize the expression quantitative trait loci and disease-associated genetic variants based on the predictions.

The principal component analysis (PCA) is used for anamoly classification and to reduce the dimensionality of micro array gene expression data and applied Stacked Auto Encoder (SAE) to categorize and segregate various cancers like acute myeloid leukemia and ovarian cancer.

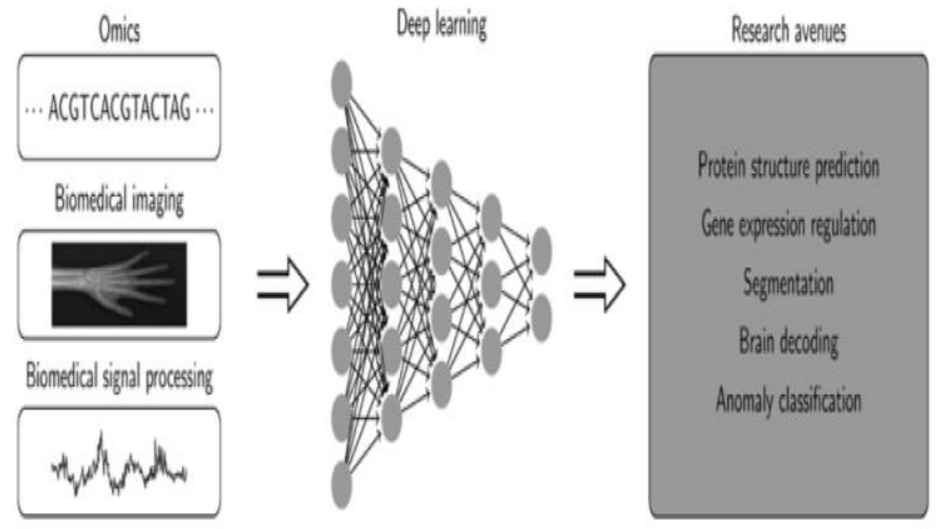

Figure 4: Implementing Deep Learning in Bioinformatics.

The above figure depicts how Deep learning is implemented in Bioinformatics. Bioinformatics is benefitted from deep learning in a large extent.

\section{DEEP LEARNING IN SPEECH RECOGNITION}

Speech recognition is a versatile and a multidisciplinary sub field of computational linguistics. It establishes procedures, methodologies and technologies that allows the perception and interpretation of spoken language into text by computers. It incorporates the understandings, knowledge and research in the linguistics, computer science and electrical engineering domains. Speech recognition has a long legacy with several waves of major innovations from the technology perspective. This field has bettered and benefited from advances in Deep learning and Big data. The worldwide industry adoption of a variety of Deep learning methods in designing and deploying speech recognition systems are the evidences for advancement. Large industries like Amazon, Google, Microsoft, Apple, IBM, Baidu, Soundhound etc have 
promoted that the significant and pivotal technology in their speech recognition systems as being based on Deep learning. A good signal processing can only lead to a top automatic speech recognition performance in defying acoustic environments. This notion leads to a unified Deep neural network framework for speech processing that can achieve both high quality enhanced speech and high accuracy automatic speech recognition [8].

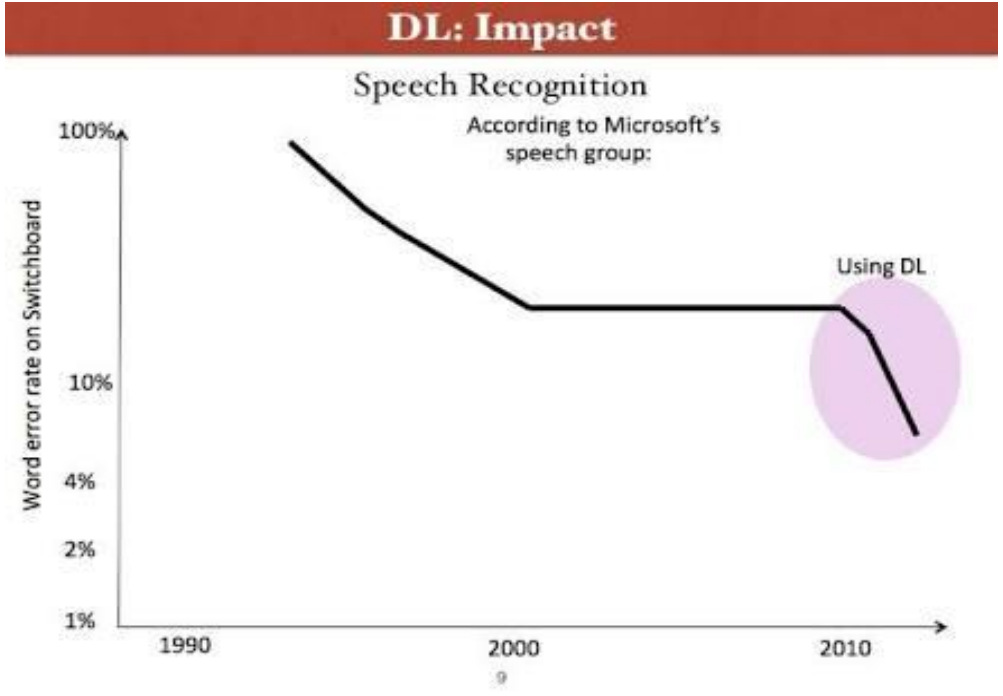

Figure 5: Portraying the Word error rate with and without Deep Learning.

The word error rate is portrayed in the above figure indicating the drop in the error rates when Deep learning is employed in speech recognition when compared to methods not involving Deep learning. The Sequence-to-sequence modelling interpolates machine translation, speech synthesis, speech recognition. Various models such as Acoustic model, Language model and Lexicons are utilized in speech recognition. Acoustic models have the likelihood of spawning observed features. Probability of any word sequence is choosen by the language models. Lexicons map words to sub-word units. The hidden Markov models are generally referred to as the acoustic models. The finest instance for the hidden Markov models is the Dynamic Bayesian network.

\section{DEEP LEARNING IN CUSTOMER RELATIONSHIP MANAGEMENT}

An approach to managing a company's interaction with current and potential customers is called as the Customer relationship management. It uses data analysis about customer's history with a company and to improve business relationships with customers. It specifically focuses on customer retention and ultimately driving sales growth. Customer Relationship Management involves Business Intelligence by incorporating information acquisition, information storage, and decision support functions. This helps to provide customized

Proceedings of the $3^{\text {rd }}$ National Conference on Image Processing, Computing, Communication, Networking and Data Analytics (NCICCNDA 2018) 
Insight into Applications of Deep Learning

customer service. It also enables customer representatives to analyze and categorize data to address customer requirements in order to provide greater customer satisfaction and retention [12]. Unchaining the data from the CRM database allows data scientists and analysts to aggregate them with other data elements. The companies can understand and learn if the customers are on the verge of leaving, product mixes and grouped predictive reordering when they perform deep analysis of their CRM data.

Customer relationship management is a demanding, popular and strategic topic in marketing and quality of service. The availability of enormous transactions data as well as processing systems has provided a great opportunity to design and predict customer behaviour [11]. Deep learning techniques can help marketing decision makers to contribute more reliable and realistic marketing strategic plans. Customer behaviour prediction model can be modelled using recurrent neural networks (RNNs) based on the client loyalty number (CLN), frequency, and monetary (RFM) variables. Deep learning methods based on RNNs are practical tools for designing and modelling time-series data.

The experiment results show that RNNs can predict RFM values of customers efficiently. This model can be later used in recommender systems for exclusive promotional offers and loyalty programs management. The fundamental framework begins with the research definition, where the aim is to use information derived from the collated data (Point of sale) to be fitted in the developed model to predict. It is followed by the data processing stage, where the collection data are cautiously examined and depurated and transformed into a method that the model would able to use and understand. This is referred to as Normalization. The concluding part of the model is the result assessment stage. From the results and outputs of the model, the best model with parameters that are befitting are attained and chosen to be the model to be executed for the prediction research for customer quantity [13].

Two classifications - Naïve bayes and Neural networks are suggested and enforced. Naïve bayes and Neural networks are distinguished with respect to specificity, accuracy and sensitivity. Weka tool is used for study purpose. Results signify that Neural networks are better than Naïve bayes method. A structure for autonomous control of a customer relationship management system has been developed and refined. Firstly, an altered version of the universally acknowledged Recency-Frequency-Monetary Value system of metrics can be used to represent the state space of clients or donors is examined. Secondly, a procedure to determine the optimal direct marketing action in discrete and continuous action space for the given individual, based on his position in the state space is described. The procedure involves the use of model-free Q-learning to train a deep neural network that relates a client's position in the state space to rewards associated with possible marketing activities. The approximated value function over the client state space can be interpreted as customer lifetime value (CLV), and thus allows for a quick plug-in estimation of CLV for a given client. Experimental results are conferred, based on Knowledge Discovery and Data Mining Tools Competition.

ISBN: 978-81-936820-0-5

Proceedings DOI: 10.21467 /proceedings. 1 


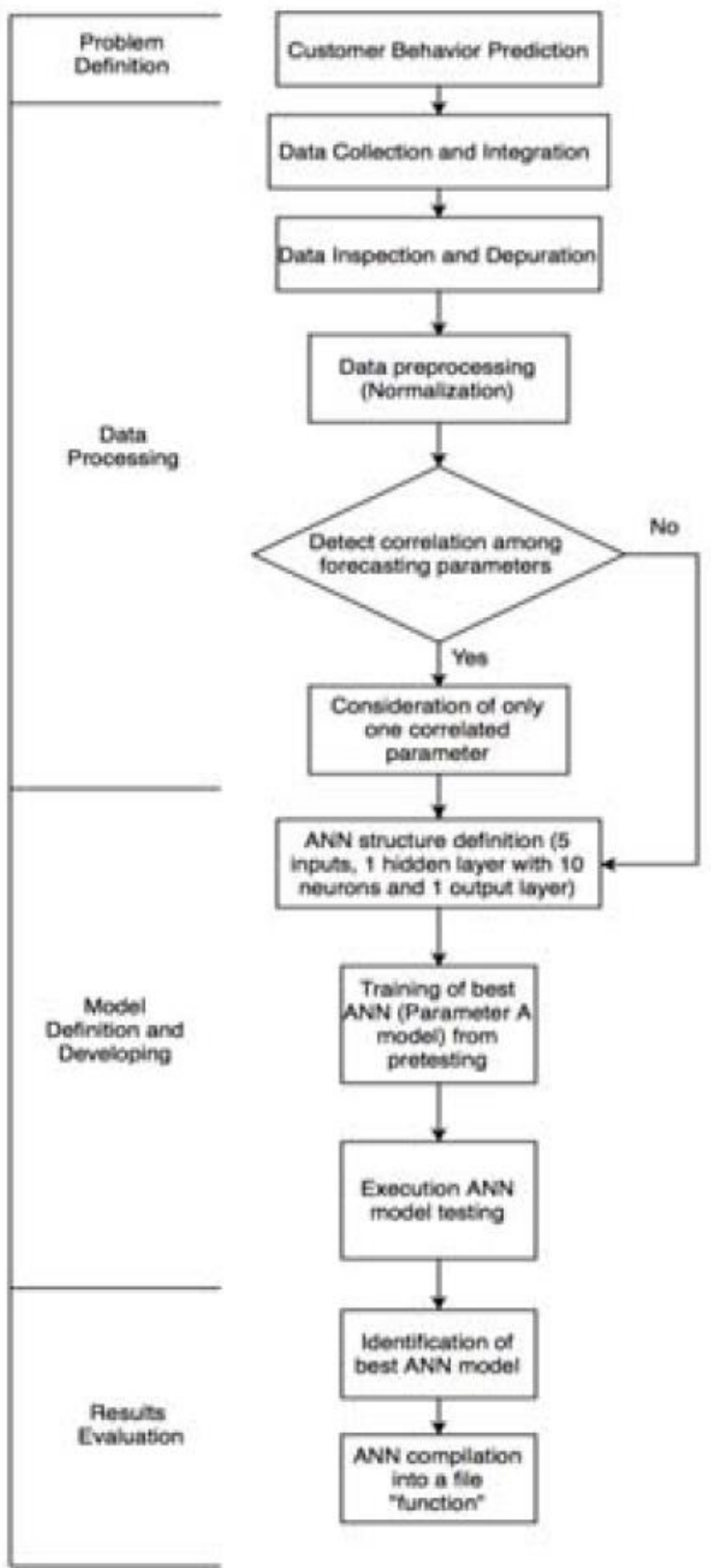

Figure 7: Deep learning-based Customer forecasting tool.

Proceedings of the $3^{\text {rd }}$ National Conference on Image Processing, Computing, Communication, Networking and Data Analytics (NCICCNDA 2018) 
Insight into Applications of Deep Learning

6 CONCLUSION

Deep learning is an impressive, quickly propelling and a proliferating discipline. It is being used today in every probable domain. It has shoved the frontier of what is possible for tasks such as Pattern recognition, image segmentation, object detection, face recognition, voice analyzing, emotion detection and gender recognition. The fuel to drive research activity and refine results to the point where deep learning solutions typically outperform even the best of human-designed pattern recognition tools is provided by Big data. More novel advancements of deep learning accompanies the capability to deal with convoluted and complicated learning tasks, thanks to the integration with different types of external memory and reinforcement learning. One striking and impressive trait of methods incorporating Deep Learning is their dexterity to accustom to data they didn't see in the training phase- a capability referred to as Generalization.

\section{REFERENCES}

[1] Kai Arulkumaran, Marc Peter Deisenroth, Miles Brundage, and Anil Anthony Bharath, "Deep reinforcement learning", 2017, IEEE.

[2] Ryo Miyajima, "Deep learning triggers a new era in industrial robotics", 2017, IEEE.

[3] Manyika J, Chui M, Brown B et al., "Big data; The next frontier for innovation, competition and production", 2011, IEEE.

[4] Dhanesh Ramachandram, Graham W. Taylor, "Deep multimodal learning”, 2017, IEEE.

[5] Farabet C, Couprie C, Najman L et al. "Learning hierarchical features fro scene labelling, pattern analysis and machine intelligence", 2013, IEEE.

[6] Seonwoo Min, Byunghan Lee, and Sungroh Yoon, 2017, Seoul national university.

[7] Bahan Zhuang, Lingqiao Liu, Yao Li, Chunhua Shen, “Attend in groups: a weakly supervised deep learning framework from learning from web data", 2017, IEEE.

[8] "An end to end deep learning approach to simultaneous speech dereverberation and acoustic modelling for robust speech recognition”, 2017, volume 11, IEEE.

[9] MinYuh Day, Yue Da Lin, "Deep learning for sentiment analysis on google play consumer review", 2017, IEEE.

[10] G. preethi, P Venkata Krishna, V savitha, "Application of Deep learning to sentiment analysis for recommender systems on cloud", 2017, IEEE.

[11] Hojjat Salehinejad and Shahryar Rahnamayan, "Customer pattern prediction: A recurrent neural network approach", 2016, IEEE.

[13] S. Ummugulthum Natchiar, "Customer relationship management classification using data mining techniques", 2014, IEEE.

[14] Kuo-Yi, Lin, Jeffrey, J.P., Tsai, “A Deep Learning based customer forecasting tool”, 2016, IEEE. 